\title{
BMJ Open Protocol for a randomised controlled trial evaluating the effects of providing essential medicines at no charge: the Carefully seLected and Easily Accessible at No Charge Medicines (CLEAN Meds) trial
}

Nav Persaud, ${ }^{1,2,3}$ Taehoon Lee, ${ }^{2}$ Haroon Ahmad, ${ }^{4}$ Winny Li, ${ }^{5}$

Michael Sergio Taglione, ${ }^{5}$ Yathavan Rajakulasingam, ${ }^{2}$ Norman Umali, ${ }^{2}$

Andrew Boozary, ${ }^{3,6}$ Richard H Glazier, ${ }^{1,2,3,7,8}$ Tara Gomes, , ${ }^{1,2,8,9,10}$

Stephen W Hwang, ${ }^{2,5,8}$ Peter Jüni, ${ }^{5,11}$ Michael Law, ${ }^{12}$

Muhammad M Mamdani, 2,5,8,9,10,13 Braden Manns, ${ }^{14,15,16,17}$ Danielle Martin, ${ }^{1,18}$

Steve Morgan, ${ }^{19}$ Paul Oh, ${ }^{20}$ Andrew David Pinto, ${ }^{1,2,3,7}$ Baiju R Shah, ${ }^{5,8}$

Frank M Sullivan, ${ }^{8,21}$ Kevin E Thorpe, ${ }^{2,7}$ Karen Tu, ${ }^{1,8,10,22,23}$ Andreas Laupacis ${ }^{1,2,8}$

To cite: Persaud N, Lee T, Ahmad $\mathrm{H}$, et al. Protocol for a randomised controlled trial evaluating the effects of providing essential medicines at no charge: the Carefully seLected and Easily Accessible at No Charge Medicines (CLEAN Meds) trial. BMJ Open 2017;7:e015686. doi:10.1136/ bmjopen-2016-015686

\section{- Prepublication history} and additional material are available. To view these files please visit the journal online (http://dx.doi.org/ 10.1136/ bmjopen-2016-015686).

Received 23 December 2016 Revised 7 April 2017 Accepted 20 April 2017

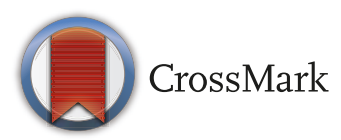

For numbered affiliations see end of article.

Correspondence to

Dr. Nav Persaud; nav.persaud@ utoronto.ca

\section{ABSTRACT}

Introduction Cost-related non-adherence to medicines is common in low-income, middle-income and highincome countries such as Canada. Medicine nonadherence is associated with poor health outcomes and increased mortality. This randomised trial will test the impact of a carefully selected list of essential medicines at no charge (compared with usual medicine access) in primary care patients reporting cost-related non-adherence.

Methods and analysis This is an open-label, parallel two-arm, superiority, individually randomised controlled trial conducted in three primary care sites (one urban, two rural) in Ontario, Canada, that was codesigned by a community guidance panel. Adult patients ( $\geq 18$ years) who report cost-related non-adherence to medicines are eligible to participate in the study. Participants will be randomised to receive free and convenient access to a carefully selected list of 125 essential medicines (based on the WHO's Model List of Essential Medicines) or usual means of medicine access. Care for patients in both groups will otherwise be unchanged. The primary outcome of this trial is adherence to appropriately prescribed medicines. Secondary outcomes include medicine adherence, appropriate prescribing, blood pressure, haemoglobin A1c, low-density lipoprotein cholesterol, patient-oriented outcomes and healthcare costs. All participants will be followed for at least 12 months.

Ethics and dissemination Ethics approval was obtained in all three participating sites. Results of the main trial and secondary outcomes will be submitted for publication in a peer-reviewed journal and discussed with members of the public and decision makers.

Trial registration number NCT02744963.

\section{Strengths and limitations of this study}

The intervention in this randomised controlled trial combines three elements: free medicine access, a carefully selected list of essential medicines, and centralised distribution and delivery of prescription medicines by a pharmacist who has access to the electronic health record.

- Medicine adherence will be measured using a combination of methods, including self-reported questionnaires, chart reviews and electronic monitoring.

- The trial participants will be recruited from primary care practices so individuals who are not already connected to a primary care provider will not be included.

- This trial will not be able to determine the contributions of the various components of the intervention towards any effects measured or the long-term effects of medicine access, including effects on hard outcomes.

\section{INTRODUCTION}

Medicine non-adherence rates range between $30 \%$ and $40 \%$ for chronic disease treatments in low-income, middle-income and high-income countries. ${ }^{1}$ The cost of medicines is one of the most important drivers of non-adherence according to observational studies and qualitative studies. ${ }^{2-4}$ Non-adherence to medicines can lead to substantial worsening of health outcomes as well as increased healthcare expenditure. ${ }^{5}$ For US 
Medicare beneficiaries, a review of 47 studies found that greater drug coverage and decreased patient cost sharing improved medicine adherence. ${ }^{6}$ Providing medicines at no charge to patients reporting cost-related non-adherence may improve medicine adherence and clinical outcomes, particularly for the elderly, vulnerable individuals and those with chronic diseases. ${ }^{67}$ There is limited literature that evaluates the impact on adherence of providing medicines at no charge. ${ }^{7}$ In the Post-Myocardial Infarction Free Rx Event and Economic Evaluation (MI FREEE) trial, which was conducted in a highly selected group of post-myocardial infarction patients and only involved antiplatelet, beta-blockers, ACE inhibitors, angiotensin receptor blockers and statins, the absolute adherence for all patients in the full coverage intervention group $(43.9 \% \pm 33.7 \%)$ was $5.4 \%$ (adjusted, 95\% CI $3.6 \%$ to $7.2 \% ; \mathrm{p}<0.001$ ) higher compared with the usual coverage control group $(38.9 \% \pm 32.7 \%){ }^{7}$ There was no significant improvement in the primary composite clinical outcome of the rate of first readmission for a major vascular event or coronary revascularisation (17.6 intervention vs 18.8 control; $\mathrm{HR}, 0.93$; $95 \%$ CI 0.82 to 1.04 ; $\mathrm{p}=0.21){ }^{7}$ However, secondary clinical outcomes of rates of total major vascular events or revascularisation (21.5 intervention vs 23.3 control; HR, $0.89 ; 95 \%$ CI 0.90 to $0.99 ; \mathrm{p}=0.03)$ and rates of first major vascular event (11.0 intervention vs 12.8 control; HR, 0.86 ; $95 \%$ CI 0.74 to $0.99 ; \mathrm{p}=0.03$ ) were significantly reduced in the full coverage group. ${ }^{7}$ In other published trials that provided access to medicines for free, the intervention differed from the control condition in ways other than free access to medicines, and participants in the control groups had access to medicines without charge. ${ }^{89}$

Adherence to inappropriately prescribed medicines can also harm people. Using 52 indicators, the prevalence of potentially inappropriate prescribing in the UK in 2007 was $27 \% .{ }^{10}$ More than $50 \%$ of US senior Medicare beneficiaries being treated with a prescription medicine received at least one potentially inappropriate medicine during a calendar year. ${ }^{11}$ There is evidence that restricted formularies improve prescribing practice and reduce inappropriate prescribing by directing prescribing based on efficacy, safety and economy. ${ }^{12-14}$ Other jurisdictions have developed short lists that include only essential medicines. Sweden's 'Wise List' of approximately 200 medicines and the UK's regional short lists are examples of successful cost-effective, evidence-based formularies in high-income countries. ${ }^{15} 16$ There are no published randomised controlled trials (RCTs) or systematic reviews that evaluate the impact of limited formularies on prescribing appropriateness in a primary care setting.

The WHO recommends that nations develop a list of essential medicines 'that satisfy the priority health care needs of the population' ${ }^{17}$ Canada is an ideal setting to study the effects of providing people with free access to an essential list of medicines because (1) cost-related non-adherence is common, (2) clinicians currently prescribe a large number of different medicines and (3) healthcare services such as seeing a doctor are generally publicly funded. In Canada, physician visits, hospitalisations, emergency department visits and some home care services are publicly insured, but the cost of medicines are only publicly insured for special populations (such as people receiving social assistance and low-income seniors). Canada is the only developed nation with universal healthcare coverage that does not include prescription medicines. The rate of cost-related non-adherence is $9.6 \%(95 \%$ CI $8.5 \%$ to $10.6 \%)$ and higher for those with low income who also have a shorter life expectancy. ${ }^{18}$ Approximately one-third $(31 \%)$ of prescriptions in the province of Quebec are not filled, and the prevalence of non-adherence is greater for more expensive medicines. ${ }^{19}$ Pharmaceutical coverage in Canada is a 'patchwork' of public and private drug plans that leaves many Canadians with inadequate or no medicine coverage. ${ }^{20}$ Public drug formularies in Canada contain thousands of products (eg, there are more than 4000 medicines on the public formulary in Ontario). In 2013, 37\% of Canadian seniors received one or more prescriptions for potentially inappropriate medicines. ${ }^{21}$ An estimated $17 \%$ of hospitalisations in Canada could be prevented if prescription drugs were used more appropriately. ${ }^{22}$ The intervention in this study could improve adherence and appropriate prescribing and ultimately improve health outcomes.

\section{AIMS AND OBJECTIVES}

The aim of this study is to evaluate the effect of providing patients recruited at primary care clinics with free and convenient access to a carefully selected set of essential medicines on appropriate medicine adherence and surrogate health outcomes:

Population - adult primary care patients who report cost-related non-adherence to medicines.

Intervention- free and convenient access to a carefully selected set of essential medicines with otherwise usual care $(\mathrm{n}=392)$.

Control- usual access to medicines and usual care ( $\mathrm{n}=392)$.

Outcomes- primary: appropriate adherence to medicines over 12 months. Secondary: appropriate prescribing, medicine adherence, haemoglobin A1c (HbA1c) levels (in patients with diabetes); blood pressure (in patients with hypertension); cholesterol (in patients with dyslipidemia); patient-oriented outcomes; and average per person medicine costs.

Participants in both groups will be treated the same except for the intervention.

\section{METHODS AND ANALYSIS \\ Design}

This will be an open-label, parallel two-arm, superiority, individually randomised controlled trial with 1:1 allocation. The trial is open label as participants are told their allocation group immediately after randomisation. 

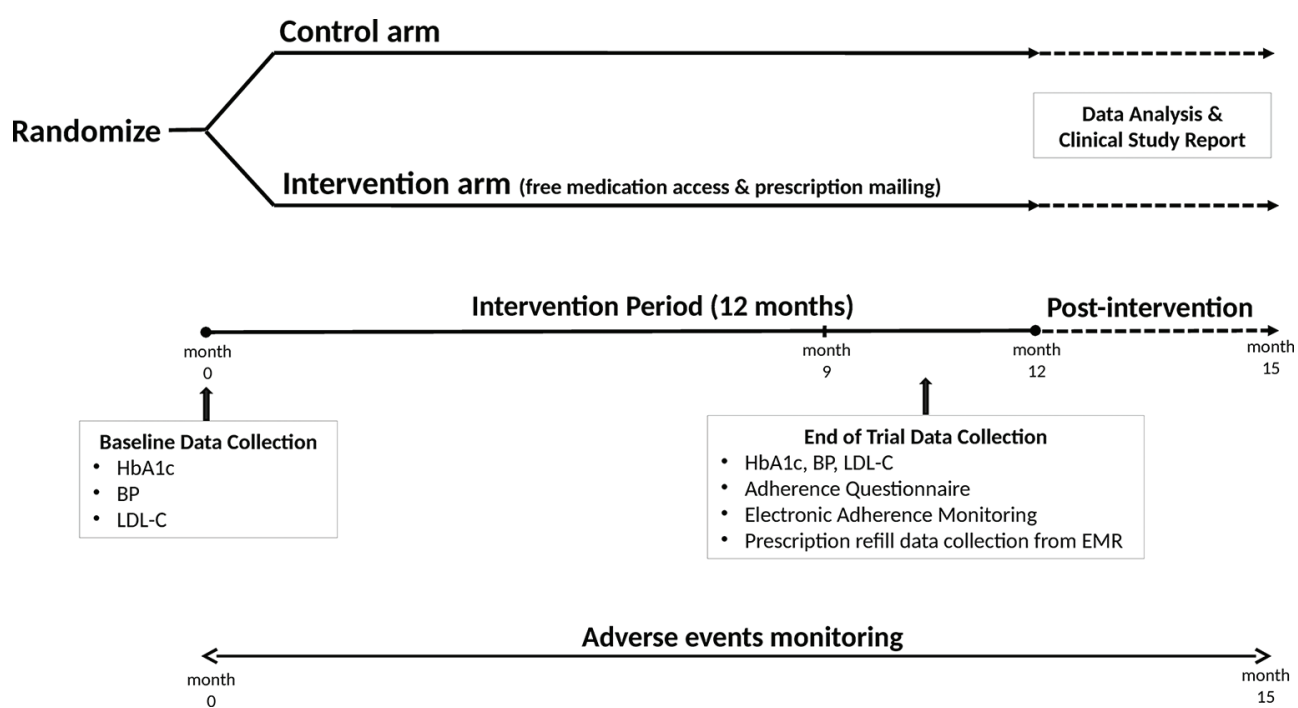

Figure 1 The design and timeline of the trial. BP, blood pressure; HbA1c, haemoglobin A1c; LDL-C, low-density lipoprotein cholesterol.; EMR, electronic medical record

Figure 1 summarises the design and timeline of the trial, and specific trial details are described below.

\section{Setting}

Carefully seLected and Easily Accessible at No Charge Medicines (CLEAN Meds) is a multicentre RCT. The study will be conducted in one urban family practice affiliated with St Michael's Hospital in Toronto (population of Toronto 2.6 million; 40000 rostered patients in practice) and two rural family practices in Ontario: Huron Shores Family Health Team in Blind River, Ontario (population 3500) and the Municipality of Assiginack Family Health Team in Manitoulin Island, Ontario (population 12000). All three sites use the same electronic medical record. These sites were selected in order to assess the intervention in both urban and rural settings.

\section{Eligibility criteria}

Patients aged 18 years or older and self-report medicine non-adherence in the last 12 months are eligible for inclusion in the study. The question used to identify non-adherence will use phrasing chosen in the Canadian Community Health Survey and similar surveys in other countries: 'In the last twelve months, did you not fill a prescription or do anything to make a prescription last longer because of the cost? ${ }^{18}$ Exclusion criteria include family member(s) living at the same address of patients already enrolled, and joined the family practice within the last 6 months. We will not exclude patients who are eligible for public medicine insurance but who do not have such coverage (eg, patients who have lost or cannot access their benefit card). We will not exclude patients with public or private medicine insurance as long as they report cost-related non-adherence. Private insurance often requires some out-of-pocket expenses, and in Canada $7 \%$ of those with prescription medicine insurance (usually through employment) still report cost-related non-adherence. ${ }^{18}$ Individuals who have private insurance and not experiencing cost-related barriers to medicine adherence will not be eligible for inclusion in the study.

\section{Randomisation and patient recruitment}

Patients will be recruited during routine primary care visits. Clinicians will briefly inform patients about the study, and interested patients will be provided with more information by research assistants. Research assistants across three sites will be responsible for patient enrolment. Patients who meet the eligibility criteria are centrally randomised into two groups: intervention group, where patients will receive free and convenient access to a carefully selected list of essential medicines, or the control group. All participants will receive usual care. Each participant will be assigned a unique patient study number. Randomisation will be concealed using a web-based tool hosted by the Applied Health Research Centre (AHRC) at the Li Ka Shing Knowledge Institute of St Michael's Hospital, and can be accessed through the electronic case report forms, which will be available through the REDCap application. The randomisation method was designed in $\mathrm{R}$ and is stratified by site using permuted blocks of varying sizes. Subsequent to the initial automated allocation, a second automated allocation will be performed, where every seventh patient enrolled into each arm across all sites (no site stratification) will receive an electronic monitoring device to measure adherence. The electronic monitoring device is based on the order of enrolment at each site. Research investigators and analysts will be blinded to treatment allocation to reduce ascertainment bias. Given the interventions, patients, clinicians and pharmacists will not be blinded to treatment allocation.

\section{Intervention arm}

Patients in the intervention arm will receive free and convenient access to a list of essential medicines (see http://www.cleanmeds.ca for a list of medicines). Both 
prescribing clinicians and intervention patients will have access to the list of essential medicines. Patients may still be prescribed other medicines not found on the essential list and access them in the usual way (eg, by paying for them).

The list of essential medicines was adapted from the 2013 WHO Model List of Essential Medicines ${ }^{23}$ using a four-step interdisciplinary, clinical peer review process. Additional medicines were added or removed from the list based on clinician suggestions, pharmaceutical industry suggestions and retrospective prescribing data. ${ }^{24}$ A panel of clinician-scientists who are free of financial conflicts of interest convenes every 3 months to evaluate the evidence and votes on recommended changes to the list using a modified nominal group technique. ${ }^{25}{ }^{26}$ For medicines removed from the list throughout the duration of the study, patients who were initially prescribed these medicines on enrolment will remain on these medicines.

Medicine dispensing for the essential medicines will primarily be through the mail. A supply of medicines that are needed for acute care (eg, antibiotics) will be stocked at each clinic study site and available for on-site dispensing by the clinician, and dispensing records will be kept to prevent contamination. For all other medicines covered by the list, the research pharmacist who will have direct access to patient electronic medical records and prescriptions will dispense these medicines as prescribed. Medicines can be delivered to a study participant within the expected geographical regions in Ontario within 1 day. Controlled substances (eg, opioids, sedatives and stimulants) will not be included in the intervention for safety reasons, and patients will access these medicines in the usual fashion and not through the research study.

After shipment, a pharmacist who will have access to interpretation services in 200 languages will call to counsel patients about their medicines. Patients without a permanent home address may choose an alternate delivery address (eg, the clinic or a support centre). Potential prescribers outside of the study sites (eg, urgent care providers or secondary care providers) will be faxed a letter with the list of essential medicines, and patients will be provided with a card with information about the list that can be shared with other providers. Both forms of communication will include contact information to reach the research pharmacist. The control group will receive standard care with their usual access to medicines and dispensing.

\section{Control arm}

Participants allocated to the control arm will have their usual access to medicines and usual care.

\section{Outcome measures}

\section{Primary outcome measure}

The primary outcome is adherence to appropriately prescribed medicines and will be determined at 12 months by assessing the percentage of prescriptions that are both (1) appropriate based on explicit criteria ${ }^{27}$ and
(2) taken as prescribed or adhered to for greater than $80 \%$ of doses.

We will use a combination of validated methods for assessing medicine adherence, which requires the assessment of both adherence and appropriateness. ${ }^{28}$ We will employ questionnaires, chart reviews and electronic monitoring as recommended and used in previous trials. ${ }^{29}$ The eight-item Morisky Medicine Adherence Scale (MMAS-8) will be completed by patients over the telephone or during a regular scheduled clinic visit between 9 and 12 months to determine the proportion of missed doses. ${ }^{30}$ Taking into consideration the number of expected medicine doses, self-reported adherence will be defined as greater than $80 \%$ (eg, one or none missed doses in 1 week for a medicine taken once a day). While questionnaires are prone to recall bias, ${ }^{28}$ because of anonymity, they are not as susceptible to social desirability bias as interviews, ${ }^{28} 29$ and do not sustain a drop in completion rates like diary reports. $^{31}$

Electronic medical record (EMR) chart reviews will also be conducted at the end of the study to identify issue dates for prescriptions and determine if chronic-use medicines are reordered when expected in a blinded fashion. Prescriptions written within 18 days of the expected renewal date (20\% of typical renewal period of 90 days) will be classified as adherent. While susceptible to pill dumping, like other objective measures such as pill counts, EMR reviews do not depend on recall. ${ }^{28}$ Electronic monitoring devices are considered by many researchers as the best available method for measuring adherence. ${ }^{32}$ These instruments passively record each time a research participant opens the pill bottle. Given the high cost of devices, only a small subset of patients in the study (112 participants, half from each treatment group) will receive them. Adherence will be defined as bottle openings to access medicines greater than $80 \%$ of the times expected. These data will be collected over the period of 12 months for chronic disease medicines.

While self-reporting tends to overestimate adherence, ${ }^{28}$ electronic monitoring is prone to underestimation. ${ }^{32}$ The combination of three measures will better approximate adherence and will be more rigorous than single measures used in previous trials. ${ }^{7-9}$

Adherence will be treated as a dichotomous variable. Participants deemed non-adherent by any of the three methods will be classified as non-adherent for the primary analysis. Since we will assume the lowest of the three possible adherence measures, we will not overestimate adherence. If data for all three measures are missing, we will assume non-adherence. We will also report adherence rates by each of the three measures for the intervention and control groups. Prescriptions for medicines intended to be taken on an 'as needed' basis (eg, analgesics, salbutamol) will be excluded from the adherence analysis.

Blinded assessors with access to patient EMR including clinician notes will assess prescribing appropriateness. Assessors will review all prescriptions written to patients in control and intervention arms using independently 
developed explicit patient-level prescribing appropriateness indicators. We will either use an existing set of appropriateness indicators ${ }^{33}$ or one based on an ongoing systematic review. ${ }^{34}$ The quality prescribing indicators relate to the management of chronic diseases such as hypertension, diabetes and asthma in a primary care setting. ${ }^{33}$ Prescribing appropriateness is a dichotomous variable and thus each prescription will be counted as appropriate or not appropriate.

\section{Secondary outcome measures}

1. proportion (\%) of prescriptions that are appropriate

2. proportion $(\%)$ of prescriptions that are adhered to

3. HbA1c levels in patients with diabetes (adjusted for baseline)

4. blood pressure in patients with hypertension (adjusted for baseline)

5. low-density lipoprotein (LDL) cholesterol levels in patients taking a statin (adjusted for baseline)

6. patient-oriented outcomes

7. healthcare costs including medicine costs.

\section{Patient-oriented outcomes}

The community guidance panel developed 'yes' or 'no' questions for study participants that will be asked between 9 and 12 months. In addition to 'yes' or 'no' responses, we will also transcribe comments.

\section{Patient-doctor relationship}

- Did you feel your care was better than before the study started? (yes or no, and open-ended)

- Did you feel your questions or concerns were addressed? (yes or no, and open-ended)

- Was your interaction with specialists different from before the study started? (yes or no, and openended)

\section{Information patients receive about medicines}

- Did the information about medicines given by the pharmacist and the doctor match? (yes or no, and comments)

- Was it easy to obtain information about the medicines? (yes or no, and comments)

- Did the information change the way you took medicines? (yes or no, and comments)

- Did you have unanswered questions about your medicines? (yes or no, and comments)

\section{Medicine delivery system}

- Was the medicine in good condition when you received it? (yes or no, and comments)

- Did you receive new medicines quickly? (only for new medicines) (yes or no, and comments)

- Did you receive the medicine before the previous prescription ran out? (only for renewals) (yes or no, and comments)
Other outcomes

- Was it easier to make ends meet at the end of the month? (yes or no, and comments)

- Do you feel your health improved? (yes or no, and comments)

- Did you have any medicine side effects? (yes or no, and comments)

- Did you have fewer healthcare visits? (yes or no, and comments)

- Is there anything else you would like to say? (yes or no, and comments)

\section{For healthcare providers}

- Ask doctor: Did you feel you had enough time in your patient encounters?

- Ask doctor: Do you think your patients are getting access to the medicines they need?

- Ask pharmacist: Was communication with the prescriber effective?

- Ask pharmacist: Was communication with the patient effective?

\section{Other outcomes}

Other outcomes that will be measured include the counts of healthcare encounters, specifically ambulatory visits, emergency department visits, hospital admission, investigations and other healthcare utilisation.

\section{Data collection}

All patients will be followed for 12 months from the date of enrolment. Medicine adherence and health outcomes data (blood pressure, HbAlc levels and cholesterol levels) will be collected during regularly scheduled appointments. Surrogate health markers captured in this trial are known to be associated with clinically important outcomes such as myocardial infarction, stroke and mortality. ${ }^{35-38}$ Inappropriate prescribing, prescription records and costs will be assessed by retrospective audits of the electronic medical record. No clinic visits are necessary for data collection. There are no differences between the two groups in the way data are collected or assessed.

\section{Statistical analysis}

Patient demographics will be summarised descriptively (eg, means and SD or median IQR for continuous variables, and frequency and percentages for categorical). Demographics will be reviewed for clinically important imbalances that may be adjusted for in a secondary analysis.

The primary analysis will be performed using an intention-to-treat principle. Appropriate adherence will be compared using a $\chi^{2}$ test, and the unadjusted treatment effect will be expressed as the absolute risk difference with 95\% CI as well as the OR with $95 \%$ CI. For the primary analysis, a two-sided $p$ value of $<0.05$ will be used to reject the null hypothesis of no difference. Adjusted analyses will employ logistic regression, and the adjusted (conditional) treatment effect will be expressed as an OR with 
95\% CI. We will perform sensitivity analyses to determine the effect of missing data. We will not adjust for site as the purpose of the study is to estimate the effect of the intervention on the population.

The first two secondary outcomes are binary at the level of prescription within patients. This will be aggregated to the patient level and logistic regression will be used to compare groups. HbA1C, blood pressure and LDL cholesterol are all continuous and measured at baseline and 9-12 months. An analysis of covariance model will be used (via linear regression) to provide the estimates of treatment effect adjusted for baseline values. Since these are all secondary analyses that are supportive or exploratory, no corrections for multiple testing will be applied and only patients with data will be included. However the intention-to-treat principle will still be followed as patients will remain allocated to their randomised groups irrespective of compliance. We will also perform a subgroup analysis to determine if the effects on medicine adherence and health outcomes differ by age and sex.

\section{Sample size rationale}

This study is powered for the primary outcome of adherence to appropriately prescribed medicines. Based on previous studies, we expect $40 \%-60 \%$ of patients in the control group will be appropriately adherent. ${ }^{7-9}$ We expect at least $90 \%$ of patients in the intervention group will be adherent to the intervention in the sense that they will agree to take at least one of the medicines (but note that adherence to the intervention is different from medicine adherence). We believe that a $10 \%$ absolute improvement in appropriate adherence (in the presence of non-compliance) is the minimal difference that is clinically important. A sample size of 392 per group is required to have a power of $80 \%$ to detect a $10 \%$ absolute difference in adherence for any control group adherence values between $40 \%$ and $60 \%$. At a two-sided type 1 error of 0.05 , no inflation for dropouts is applied to the sample size calculation since dropouts will be considered non-adherent. Based on previous trials, we expect the dropout rate to be approximately $5 \% .^{7-9}$

\section{Monitoring}

\section{Data and Safety Monitoring Board}

The Data and Safety Monitoring Board (DSMB) will meet every 3 months. The primary purpose of the DSMB will be to ensure medicine incidents are properly addressed. Each medicine error will be reported to the DSMB immediately. The DSMB will make recommendations to the research team about how to mitigate the harm from the medicine incidents and how to prevent future similar errors. The DSMB will have the power to recommend discontinuation of the trial if there is an excess of medicine incidents or if identified incidents are not appropriately managed.
Adverse events

Monitoring for medicine incidents and adverse drug reactions will occur from the point of enrolment into the study, and will continue for 3 months after the completion of the 12-month study period for each participant. The risk of medicine incidents will be mitigated by having the research pharmacist review the EMR of intervention patients before initiating or transitioning to alternate treatments. We will provide clinicians with instructions about how to manage patients who experience discontinuation effects (eg, from antidepressants). Discontinuation effects will be reported as medicine adverse effects. Medicine incidents and serious adverse events will be collected in the electronic case report form, and each event will be reported to the DSMB, who will assess ongoing safety of the intervention.

\section{Management}

The research team will receive trial implementation assistance from the AHRC. Study data and patient questionnaires will be entered and maintained on a secure password-protected database developed using REDCap (https://www.project-redcap.org/) and will be accessible via the internet for data entry purposes. Corrections or changes in the data management system will be tracked with the retention of the original data and the corrected data with the date of data entry and submitting personnel.

We expect enrolment to be complete in 12 months, and each participant will be followed for 12 months plus an additional 3 months of follow-up for adverse events. So the study should be completed approximately 27 months after the first patient is enrolled.

\section{Public involvement}

The trial intervention and some outcomes were codesigned by a panel of 11 community members who were recruited via canvassing, random digit dialling and publicly posting notices. These individuals met with the research team monthly starting more than 6 months before the study started. These meetings will continue during the study.

\section{Ethics and dissemination}

Ethics approval for the conduct of this study was obtained from the St Michael's Research Ethics Board, the Huron Shores Family Health Team Research Ethics Committee and the Laurentian University Research Ethics Board. Version 3.0, which was dated 18 March 2015, of the protocol was approved. Any important protocol modifications will be made after communication with the research ethics boards and will be described in the resulting publications. Informed consent will be obtained by research assistants (see online supplementary appendix 1 consent form). Personal health data are maintained in the electronic health record. All investigators will have access to the final trial data set.

There are no restrictions on dissemination of the results, and we plan to disseminate the results of the study 
to participants, healthcare providers and governments. We plan to make participant-level data publicly available when the results are published in a peer-reviewed journal.

The trial is registered with clinicaltrials.gov NCT02744963.

\section{SUMMARY}

The CLEAN Meds trial will be the first randomised study to evaluate the impact of providing primary care patients with free access to a list of essential medicines using a centralised mail-order dispensing method where the pharmacist has access to the electronic health record. This study is powered to detect differences in important clinical outcomes in addition to medicine adherence, prescribing appropriateness and healthcare costs. The findings may inform public policy decisions around medicine access in Canada and other countries.

Strengths of this study include the fact that a wide range of medicines will be provided to patients with different needs as would happen if public policy were changed, participants are recruited from both urban and rural sites, and there are few exclusion criteria or requirements on participants. Limitations of the study include the use of surrogate health outcomes, the inability to disentangle the effects of the components of the intervention, the lack of long-term intervention provisions and follow-up, insufficient power to detect smaller $(<10 \%)$ improvements in adherence, and the lengthy informed consent form that may discourage some eligible individuals from participating.

\section{FEASIBILITY}

We enrolled 323 participants after 26 weeks.

\section{Author affiliations}

${ }^{1}$ Department of Family and Community Medicine, St Michael's Hospital, Toronto, Canada

${ }^{2}$ Li Ka Shing Knowledge Institute, St Michael's Hospital, Toronto, Ontario, Canada

${ }^{3}$ Department of Family and Community Medicine, University of Toronto, Toronto, Ontario, Canada

${ }^{4}$ Department of Medicine, McMaster University, Hamilton, Ontario, Canada

${ }^{5}$ Department of Medicine, University of Toronto Faculty of Medicine, Toronto, Ontario, Canada

${ }^{6}$ Department of Health Policy and Management, Harvard School of Public Health, Boston, Massachusetts, United States

${ }^{7}$ Dalla Lana School of Public Health, University of Toronto, Toronto, Ontario, Canada

${ }^{8}$ Institute for Clinical Evaluative Sciences, Toronto, Canada

${ }^{9}$ Leslie Dan Faculty of Pharmacy, University of Toronto, Toronto, Canada

${ }^{10}$ Institute of Health Policy, Management, and Evaluation, University of Toronto,

Toronto, Canada

${ }^{11}$ Applied Health Research Centre, Li Ka Shing Knowledge Institute, St Michael's Hospital, Toronto, Canada

${ }^{12}$ Centre for Health Services and Policy Research, School of Population and Public

Health, University of British Columbia, Vancouver, British Columbia, Canada

${ }^{13}$ Centre for Healthcare Analytics Research and Training, St Michael's Hospital,

Toronto, Canada

${ }^{14}$ Department of Community Health Science, Cumming School of Medicine, University of Calgary, Calgary, Canada

${ }^{15}$ Department of Medicine, Cumming School of Medicine, University of Calgary, Calgary, Canada
${ }^{16}$ Interdisciplinary Chronic Disease Collaboration, Cumming School of Medicine, University of Calgary, Calgary, Canada

${ }^{17}$ Libin Cardiovascular Institute, Cumming School of Medicine, University of Calgary, Calgary, Canada

${ }^{18}$ Women's College Hospital Institute for Health Systems Solutions and Virtual Care, Women's College Hospital, Toronto, Canada

${ }^{19}$ Centre for Health Services and Policy Research, University of British Columbia, Vancouver, Canada

${ }^{20}$ Toronto Rehabilitation Institute, University Health Network, Toronto, Canada

${ }^{21}$ Department of Research and Innovation, North York General Hospital, Toronto, Canada

${ }^{22}$ Central Primary Care and Population Health Research Program, Institute for Clinical Evaluative Sciences, Toronto, Canada

${ }^{23}$ Toronto Western Hospital Family Health Team, University Health Network, Toronto, Canada

Correction notice This paper has been amended since it was published Online First. Owing to a scripting error, some of the publisher names in the references were replaced with 'BMJ Publishing Group'. This only affected the full text version, not the PDF. We have since corrected these errors and the correct publishers have been inserted into the references.

Acknowledgements NP, RHG and ADP were supported as Clinician Scientists by the Department of Family and Community Medicine at the University of Toronto and at St Michael's Hospital. NP is supported by a fellowship from Physicians Services Incorporated Foundation. KT was supported as a Clinician Scientist by the Department of Family and Community Medicine at the University of Toronto and at Toronto Western Hospital. ML received salary support through a Canada Research Chair and a Michael Smith Foundation for Health Research Scholar Award.

Contributors All authors contributed substantially to conception and the design of the protocol, all revised the manuscript critically for important intellectual content, all approved the final manuscript, and all are accountable for all aspects of the work in ensuring that questions related to the accuracy or integrity of any part of the work are appropriately investigated and resolved.

Funding The trial is funded by the Ontario SPOR Support Unit and the Canadian Institutes of Health Research.

Competing interests None declared.

Patient consent The protocol does not contain personal medical information about an identifiable living individual and does not require patients' explicit consent.

Ethics approval Ethics approval for the conduct of this study was obtained from the St Michaels Research Ethics Board, the Huron Shores Family Health Team Research Ethics Committee and the Laurentian University Research Ethics Board.

Provenance and peer review Not commissioned; externally peer reviewed.

Open Access This is an Open Access article distributed in accordance with the Creative Commons Attribution Non Commercial (CC BY-NC 4.0) license, which permits others to distribute, remix, adapt, build upon this work non-commercially, and license their derivative works on different terms, provided the original work is properly cited and the use is non-commercial. See: http://creativecommons.org/ licenses/by-nc/4.0/

(c) Article author(s) (or their employer(s) unless otherwise stated in the text of the article) 2017. All rights reserved. No commercial use is permitted unless otherwise expressly granted.

\section{REFERENCES}

1. Bowry AD, Shrank WH, Lee JL, et al. A systematic review of adherence to cardiovascular medications in resource-limited settings. J Gen Intern Med 2011;26:1479-91.

2. Laba TL, Essue B, Kimman M, et al. Understanding patient Preferences in Medication Nonadherence: a Review of Stated Preference Data. Patient 2015;8:385-95.

3. Lexchin J, Grootendorst P. Effects of prescription drug user fees on drug and health services use and on health status in vulnerable populations: a systematic review of the evidence. Int J Health Serv 2004;34:101-22.

4. Goldman DP, Joyce GF, Zheng Y. Prescription drug cost sharing: associations with medication and medical utilization and spending and health. JAMA 2007;298:61-9. 
5. Osterberg L, Blaschke T. Adherence to medication. N Engl J Med 2005;353:487-97.

6. Shenolikar R, Bruno AS, Eaddy M, et al. Sensitivity of medication use to formulary controls in medicare beneficiaries: a review of the literature. Am Health Drug Benefits 2011;4:465-74.

7. Choudhry NK, Avorn J, Glynn RJ, et al. Full coverage for preventive medications after myocardial infarction. $N$ Engl J Med 2011;365:2088-97.

8. Thom S, Poulter N, Field J, et al. Effects of a fixed-dose combination strategy on adherence and risk factors in patients with or at high risk of CVD: the UMPIRE randomized clinical trial. JAMA 2013;310:918-29.

9. Farooq S, Nazar Z, Irfan M, et al. Schizophrenia medication adherence in a resource-poor setting: randomised controlled trial of supervised treatment in out-patients for schizophrenia (STOPS). Br J Psychiatry 2011;199:467-72.

10. Bradley MC, Motterlini N, Padmanabhan S, et al. Potentially inappropriate prescribing among older people in the United Kingdom. BMC Geriatr 2014;14:72.

11. Jirón M, Pate V, Hanson LC, et al. Trends in prevalence and determinants of potentially inappropriate prescribing in the United States: 2007 to 2012. J Am Geriatr Soc 2016;64:788-97.

12. King MA, Roberts MS. The influence of the Pharmaceutical Benefits Scheme (PBS) on inappropriate prescribing in australian nursing homes. Pharm World Sci 2007;29:39-42.

13. Feely J, Chan R, Cocoman L, et al. Hospital formularies: need for continuous intervention. BMJ 1990;300:28-30.

14. Yakabowich MR, Keeley G, Montgomery PR. Impact of a formulary on personal care homes in Manitoba. CMAJ 1994;150:1601-7.

15. Gustafsson LL, Wettermark B, Godman B, et al. The 'wise list'- a comprehensive concept to select, communicate and achieve adherence to recommendations of essential drugs in ambulatory care in Stockholm. Basic Clin Pharmacol Toxicol 2011;108:224-33.

16. Hill-Smith I. Sharing resources to create a district drug formulary: a countywide controlled trial. Br J Gen Pract 1996;46:271-5.

17. World Health Organization. The selection of essential drugs: report of the World Health Organization Expert Committee. Geneva: World Health Organization, 1977. http://apps.who.int/iris/bitstream/10665/ 41272/1/WHO_TRS_615.pdf. (accessed 16 Dec 2016).

18. Law MR, Cheng L, Dhalla IA, et al. The effect of cost on adherence to prescription medications in Canada. CMAJ 2012;184:297-302.

19. Tamblyn R, Eguale T, Huang A, et al. The incidence and determinants of primary nonadherence with prescribed medication in primary care: a cohort study. Ann Intern Med 2014;160:441-50.

20. Kapur V, Basu K. Drug coverage in Canada: who is at risk? Health Policy 2005;71:181-93.

21. Morgan SG, Hunt J, Rioux J, et al. Frequency and cost of potentially inappropriate prescribing for older adults: a cross-sectional study. CMAJ Open 2016;4:E346-E351.
22. Samoy LJ, Zed PJ, Wilbur K, et al. Drug-related hospitalizations in a tertiary care internal medicine service of a canadian hospital: a prospective study. Pharmacotherapy 2006;26:1578-86.

23. World Health Organization,. WHO model list of essential medicines: 18th list, 2013. Available from. http://apps.who.int/iris/bitstream/ 10665/93142/1/EML_18_eng.pdf?ua=1. (accessed 16 Dec 2016).

24. Taglione MS, Ahmad H, Slater M, et al. Development of a preliminary essential medicines list for Canada. CMAJ Open 2017;5:E137-E143.

25. Nair R, Aggarwal R, Khanna D. Methods of formal consensus in classification/diagnostic criteria and guideline development. Semin Arthritis Rheum 2011;41:95-105.

26. Guyatt GH, Oxman AD, Vist GE, et al. GRADE: an emerging consensus on rating quality of evidence and strength of recommendations. BMJ 2008;336:924-6.

27. Fernández Urrusuno R, Pérez Pérez P, Montero Balosa MC, et al . Compliance with quality prescribing indicators linked to financial incentives: what about not incentivized indicators?: an observational study. Eur J Clin Pharmacol 2014;70:303-11.

28. Lehmann A, Aslani P, Ahmed R, et al. Assessing medication adherence: options to consider. Int J Clin Pharm 2014;36:55-69.

29. Garber MC, Nau DP, Erickson SR, et al. The concordance of selfreport with other measures of medication adherence: a summary of the literature. Med Care 2004;42:649-52.

30. Morisky DE, Ang A, Krousel-Wood M, et al. Predictive validity of a medication adherence measure in an outpatient setting. $J$ Clin Hypertens 2008;10:348-54.

31. Butz AM, Donithan M, Bollinger ME, et al. Monitoring nebulizer use in children: comparison of electronic and asthma diary data. Ann Allergy Asthma Immunol 2005;94:360-5.

32. Cook P, Schmiege S, McClean M, et al. Practical and analytic issues in the electronic assessment of adherence. West $J$ Nurs Res 2012;34:598-620.

33. Fernández Urrusuno R, Montero Balosa MC, Pérez Pérez $\mathrm{P}$, et al. Compliance with quality prescribing indicators in terms of their relationship to financial incentives. Eur J Clin Pharmacol 2013;69:1845-53.

34. Persaud P, Bali A, Rajakulasingam Y, et al. Explicit individual patient indicators of potentially inappropriate prescribing: a systematic review.PROSPERO: International prospective register of systematic reviews. 2016 https://www.crd.york.ac.uk/PROSPERO/display_ record.asp?ID=CRD42016051037.

35. Macisaac RJ, Jerums G. Intensive glucose control and cardiovascular outcomes in type 2 diabetes. Heart Lung Circ 2011;20:647-54.

36. Nagai M, Kario K. Visit-to-visit blood pressure variability, silent cerebral injury, and risk of stroke. Am J Hypertens 2013;26:1369-76.

37. Briasoulis A, Agarwal V, Tousoulis D, et al. Effects of antihypertensive treatment in patients over 65 years of age: a meta-analysis of randomised controlled studies. Heart 2014;100:317-23.

38. Ridker PM, Cook NR. Statins: new american guidelines for prevention of cardiovascular disease. Lancet 2013;382:1762-5. 